\title{
Shape and change point analyses of the Birnbaum-Saunders- $t$ hazard rate and associated estimation
}

\author{
Cecilia Azevedo ${ }^{\mathrm{a}}$ Víctor Leiva ${ }^{\mathrm{b}, *}$ Emilia Athayde ${ }^{\mathrm{c}}$ \\ N. Balakrishnan ${ }^{\mathrm{d}}$ \\ ${ }^{a}$ Centro de Matemática, Universidade do Minho, Portugal \\ ${ }^{\mathrm{b}}$ Departamento de Estadística, Universidad de Valparaíso, Chile \\ ${ }^{\mathrm{c}}$ Departamento de Matemática e Aplicações, Universidade de Minho, Portugal \\ ${ }^{\mathrm{d}}$ Department of Mathematics and Statistics, McMaster University, Canada
}

\begin{abstract}
The hazard rate is a statistical indicator commonly used in lifetime analysis. The Birnbaum-Saunders (BS) model is a life distribution originated from a problem pertaining to material fatigue that has been applied to diverse fields. The BS model relates the total time until failure to some type of cumulative damage that is normally distributed. The generalized BS (GBS) distribution is a class of positively skewed models with lighter and heavier tails than the BS distribution. Particular cases of GBS distributions are the BS and BS-Student- $t$ (BS- $t$ ) models. In this paper, we discuss shape and change point analyses for the hazard rate of the BS- $t$ distribution. In addition, we evaluate the performance of the maximum likelihood and moment estimators of this change point using Monte Carlo methods. We also present an application with a real life data set useful for survival analysis, which shows the convenience of knowing such instant of change for establishing a reduction in the dose and, as a consequence, in the cost of the treatment.
\end{abstract}

Key words: Failure rate; Generalized Birnbaum-Saunders distribution; Lifetime data; Likelihood methods; Moment estimation; Monte Carlo methods.

Mathematics Subject Classification: Primary 60E05; Secondary 62N05.

\section{Introduction}

A useful indicator for lifetime analysis is the hazard or failure rate (FR). The FR can be increasing (IFR class), decreasing (DFR class) or constant. However, there are distributions that have a non-monotone FR. In that case, an important value for the FR is its change point. Within the class of distributions with a non-monotone FR, we can identify bathtub (BT) or inverse bathtub (IBT) behaviors. Particularly, for the class of IBT-shaped FRs, the FR can be initially increasing until its change point and then decreasing to zero or it becomes stabilized at a positive constant (not at zero). For this reason, for distributional families with a non-monotone $\mathrm{FR}$, its change point and its limiting behavior are aspects important and useful to study.

\footnotetext{
* Corresponding author. Víctor Leiva. Departamento de Estadística, Universidad de Valparaíso, Avda. Gran Bretaña 1111, Playa Ancha, Valparaíso, Chile. URL: www.deuv.cl/leiva

Email address: victor. leiva@uv.cl (Víctor Leiva).
} 
It is well-known that inference upon normality is vulnerable to atypical data. In lifetime data, in addition to the presence of atypical cases, the distribution of this type of data is often skewed. For more details about lifetime analysis, the reader can refer to Lawless (2002), Marshall and Olkin (2007), and Saunders (2007).

The Birnbaum-Saunders (BS) life distribution has recently received a considerable attention. A comprehensive treatment on this distribution can be found in Birnbaum and Saunders (1969), Johnson et al. (1995, pp. 651-662) and Athayde et al. (2012). The BS distribution has been applied in engineering and in several other areas, such as business, environment, industry, medicine, psychology and toxicology; see Balakrishnan et al. (2007, 2009a), Leiva et al. (2007, 2008b, 2009, 2010a,b, 2011a,b), Cysneiros et al. (2008), Barros et al. (2008), Guiraud (2009), Bhatti (2010), Kotz et al. (2010), Cordeiro and Lemonte (2011), Vilca et al. (2010, 2011), Vanegas et al. (2012), Cancho et al. (2012) and Paula et al. (2012). Studies on the BS FR can be found in Kundu et al. (2008) and Bebbington et al. (2008). In spite of the interest on the BS distribution, it also presents parameter estimates that are sensitive to atypical data.

Díaz-García and Leiva (2005) generalized the BS distribution to obtain a major degree of flexibility essentially in the kurtosis; see also Vilca and Leiva (2006), Sanhueza et al. (2008), Barros et al. (2009) and Balakrishnan et al. (2009b) for some further discussions in this direction. The generalized BS (GBS) family has as particular cases the BS-classic and BS-Student- $t$ distributions. The BS- $t$ distribution can be considered in place of the BS distribution to produce parameter estimates that are less sensitive to atypical data; see Leiva et al. (2008a), Barros et al. (2008) and Paula et al. (2012). Once the parameters of the BS- $t$ distribution are estimated, we can use the invariance property of the maximum likelihood (ML) estimators for determining the BS- $t$ FR and its change point. Alternatively, the moment estimation method can also be used; see Leiva et al. (2008a,b).

The main aims of this work are (i) to present a mathematical study of the shape and change point of the BS- $t$ FR; (ii) to computationally evaluate the performance of the ML and moment estimators of this FR using Monte Carlo methods; and (iii) to carry out a real lifetime data analysis.

The paper proceeds as follows. Section 2 contains a background about BS and BS- $t$ distributions. Section 3 presents a study of the shape of the BS- $t$ FR following the lines of the works by Kundu et al. (2008) and Bebbington et al. (2008). Section 4 describes the numerical part of this work. This part includes a simulation study on the ML and moment estimates of the change point of the BS- $t$ FR and an illustration with real life data. Finally, some concluding remarks are made in Section 5.

\section{Preliminary details on BS and BS- $t$ distributions}

If a random variable (RV) $T$ follows a BS distribution with shape and scale parameters $\alpha>0$ and $\beta>0$, respectively, the notation $T \sim \operatorname{BS}(\alpha, \beta)$ is used. Here, $\beta$ is also the median of the distribution. BS and standard normal RVs are related by

$$
T=\beta\left[\frac{\alpha Z}{2}+\sqrt{\left\{\frac{\alpha Z}{2}\right\}^{2}+1}\right]^{2} \sim \mathrm{BS}(\alpha, \beta) \quad \text { and } \quad Z=\frac{1}{\alpha}\left[\sqrt{\frac{T}{\beta}}-\sqrt{\frac{\beta}{T}}\right] \sim \mathrm{N}(0,1),
$$

such that $W=Z^{2}=[T / \beta+\beta / T-2] / \alpha^{2} \sim \chi^{2}(1)$. The probability density (PDF) and cumulative distribution (CDF) functions of $T$ are $f_{T}(t)=\phi(A(t ; \alpha, \beta)) A^{\prime}(t ; \alpha, \beta)$ and 
$F_{T}(t)=\Phi(A(t ; \alpha, \beta))$, for $t>0$, respectively, where $\Phi(\cdot)$ is the $\mathrm{N}(0,1)$ CDF defined as $\Phi(z)=\int_{-\infty}^{z} \phi(t) d t$, with $\phi(z)=\mathrm{d} \Phi(z) / \mathrm{d} z=\exp \left(-z^{2} / 2\right) / \sqrt{2 \pi}$, for $z \in \mathbb{R}$, being the corresponding standard normal PDF and

$$
A(x ; a, b)=\frac{1}{a}\left[\sqrt{\frac{x}{b}}-\sqrt{\frac{b}{x}}\right] \text { and } A^{\prime}(x ; a, b)=\frac{\mathrm{d} A(x ; a, b)}{\mathrm{d} x}=\frac{1}{2 a b}\left[\left\{\frac{x}{b}\right\}^{-\frac{1}{2}}+\left\{\frac{x}{b}\right\}^{-\frac{3}{2}}\right] .
$$

The BS distribution possesses the following properties: $c T \sim \operatorname{BS}(\alpha, c \beta)$, with $c>0$, and $1 / T \sim \mathrm{BS}(\alpha, 1 / \beta)$. The $q$ th and $[1-q]$ th quantiles of $T$ are, respectively,

$$
t_{q}=\frac{\beta}{4}\left[\alpha z_{q}+\sqrt{\alpha^{2} z_{q}^{2}+4}\right]^{2} \text { and } t_{1-q}=\frac{\beta}{4}\left[\alpha z_{q}-\sqrt{\alpha^{2} z_{q}^{2}+4}\right]^{2}, 0<q<1,
$$

where $z_{q}$ is the $\mathrm{N}(0,1) q$ th quantile and $t_{q}=F_{T}^{-1}(q)$, with $F_{T}^{-1}(\cdot)$ being the inverse CDF of $T$.

Similarly, if a RV T follows a BS- $t$ distribution with shape parameters $\alpha>0$ and $\nu>0$ ( $\nu$ is also known as degrees of freedom), and scale parameter $\beta>0$, then we use the notation $T \sim \operatorname{BS}-t(\alpha, \beta ; \nu)$. In this case, the $\operatorname{RVs} T$ and $Z$ are related as

$$
T=\frac{\beta}{4}\left[\alpha Z+\sqrt{\alpha^{2} Z^{2}+4}\right]^{2} \sim \operatorname{BS}-t(\alpha, \beta ; \nu) \quad \text { and } \quad Z=\frac{1}{\alpha}\left[\sqrt{\frac{T}{\beta}}-\sqrt{\frac{\beta}{T}}\right] \sim \mathrm{t}(\nu),
$$

so that now $W=Z^{2}=[T / \beta+\beta / T-2] / \alpha^{2} \sim \mathcal{F}(1, \nu)$. Hence, the PDF and CDF of $T$ are $f_{T}(t ; \nu)=\phi_{t}(A(t ; \alpha, \beta) ; \nu) A^{\prime}(t ; \alpha, \beta)$ and $F_{T}(t ; \nu)=\Phi_{t}(A(t ; \alpha, \beta) ; \nu)$, for $t>0$, respectively, where $\Phi_{t}(\cdot ; \nu)$ is the Student- $t$ CDF defined by

$$
\Phi_{t}(z ; \nu)=\frac{1}{2}\left[1+\mathrm{I}_{\frac{z^{2}}{z^{2}+\nu}}\left(\frac{1}{2}, \frac{1}{2} \nu\right)\right], \text { with } \phi_{t}(z ; \nu)=\frac{\mathrm{d} \Phi_{t}(z ; \nu)}{\mathrm{d} z}=\frac{\Gamma\left(\frac{\nu+1}{2}\right)}{\sqrt{\nu \pi} \Gamma\left(\frac{\nu}{2}\right)}\left[1+\frac{z^{2}}{\nu}\right]^{-\frac{\nu+1}{2}}
$$

for $z \in \mathbb{R}$ and $\nu>0$, with $\phi_{t}(\cdot ; \nu)$ denoting the Student- $t$ PDF, $\mathrm{I}_{x}(a, b)=\int_{0}^{x} t^{a-1}[1-$ $t]^{b-1} \mathrm{~d} t / \int_{0}^{1} t^{a-1}[1-t]^{b-1} \mathrm{~d} t$ being the incomplete beta function ratio and $A(\cdot ; \alpha, \beta)$ given as in (2). The BS- $t$ distribution has the same properties as the BS distribution, whereas the $q$ th and $[1-q]$ th quantiles of $T$ are as in (3), where now $z_{q}$ is the $t(\nu) q$ th quantile.

\section{Shape analysis of the BS- $t$ hazard rate}

The FR is a useful indicator for diverse practical applications. In addition. the FR allows us to characterize the behavior of statistical distributions. A misspecification of the FR could have serious consequences in the estimation procedure; see, e.g., Bhatti (2010). The $\mathrm{FR}$ of a RV $T$ is given by $h_{T}(t)=f_{T}(t) /\left[1-F_{T}(t)\right]$, for $t>0$ and $0<F_{T}(t)<1$, where $f_{T}(\cdot)$ and $F_{T}(\cdot)$ are the $\mathrm{PDF}$ and $\mathrm{CDF}$ of $T$, respectively.

\subsection{TTT curve}

The FR of a RV T can be characterized by the scaled total time on test (TTT) function given by $W_{T}(u)=H_{T}^{-1}(u) / H_{T}^{-1}(1)$, for $0 \leq u \leq 1$, where $H_{T}^{-1}(u)=\int_{0}^{F_{T}^{-1}(u)}\left[1-F_{T}(y)\right] d y$, with $F_{T}^{-1}(\cdot)$ being again the inverse $\mathrm{CDF}$ of $T$. Now, $W_{T}(\cdot)$ can be empirically approximated allowing to construct the empirical scaled TTT curve by plotting the consecutive points $\left[k / n, W_{n}(k / n)\right]$, where $W_{n}(k / n)=\sum_{i=1}^{k} T_{(i)}+[n-k] T_{(k)} / \sum_{i=1}^{n} T_{(i)}$, for $k=0, \ldots, n$, with $T_{(i)}$ being the corresponding $i$ th order statistic. By means of the empirical scaled TTT 
curve, we can detect the type of FR that the lifetime data have and, as a consequence, the type of distribution that would be suitable to model the data with. We can observe from Figure 1 different theoretical shapes for the scaled TTT curve; for more details about the TTT method, see Aarset (1987). Thus, a TTT curve that is concave (or convex) corresponds to the IFR (or DRF) class. A concave (or convex) and then convex (or concave) shape for the TTT curve corresponds to a IBT (or BT) FR. A TTT plot expressed by a straight line is an indication that the exponential distribution must be used.

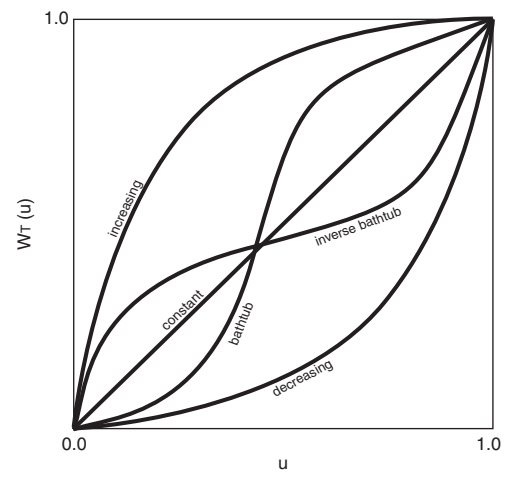

Fig. 1. Scaled TTT curves for distributions with the indicated FR shape.

\subsection{BS-t hazard rate}

The gamma and Weibull distributions belong to both IFR and DFR classes. The lognormal (LN) distribution has a non-monotone FR, because it is initially increasing until its change point and then decreasing to zero. For the BS FR, we have: (i) it behaves similarly to the LN FR, but it decreases until it becomes stabilized at a positive constant (not at zero); see Nelson (1990, p. 70); (ii) its behavior is similar to that of the inverse Gaussian FR; see Chhikara and Folks (1989, p. 153); and (iii) its average is nearly non-decreasing (close to the IFRA class); see Birnbaum and Saunders (1969). Fatigue data have usually a unimodal FR, which also occurs with environmental, financial duration and survival data; see Kundu et al. (2008), Bhatti (2010) and Vilca et al. (2010);

Let $T \sim \operatorname{BS}(\alpha, \beta)$. Then, $h_{T}(t)=\phi(A(t ; \alpha, \beta)) A^{\prime}(t ; \alpha, \beta) / \Phi(-A(t ; \alpha, \beta))$, for $t>0$. It is possible to note that $h_{T}(t)$ is unimodal for any $\alpha$, being increasing for $t<t_{c}$ and decreasing for $t>t_{c}$, with $t_{c}$ denoting its change point. In addition, $h_{T}(t)$ approaches $1 /\left[2 \alpha^{2} \beta\right]$ as $t \rightarrow \infty$. Moreover, $h_{T}(t)$ tends to be increasing as $\alpha \rightarrow 0$; see Chang and Tang (1993). Thus, it is of interest to know from which value of $\alpha$ the FR of $T$ is nearly IFR. Although it is not possible to obtain an analytical result to answer it, a numerical study indicates that the BS distribution is close to the IFR family when $\alpha<0.41$ and $0<t<8 \beta$, which implies an IFRA class. Also, Birnbaum and Saunders (1969) showed by numerical computations that the average FR of $T$ decreases slowly for $t<1.64$.

Let $T \sim \mathrm{BS}-t(\alpha, \beta ; \nu)$. Then, $h_{T}(t ; \nu)=\phi_{t}(A(t ; \alpha, \beta) ; \nu) A^{\prime}(t ; \alpha, \beta) / \Phi_{t}(-A(t ; \alpha, \beta) ; \nu)$, for $t>0$. Thus, we have: (R1) $\lim _{t \rightarrow 0} h_{T}(t ; \nu)=\lim _{t \rightarrow 0} f_{T}(t ; \nu)$ and equals (i) $\infty$ if $\nu=1$,(ii) $\alpha^{2} /[2 \beta]$ if $\nu=2$, and (iii) 0 if $\nu>2$; and (R2) let $\eta(t)=-f_{T}^{\prime}(t) / f_{T}(t)$ be Glaser's function associated with a $\operatorname{PDF} f_{T}(\cdot)$ of a positive RV $T$ such that this PDF does not vanish anywhere on $(0, \infty)$. Then, if $f_{T}(\cdot)$ is twice differentiable with corresponding $\mathrm{FR} h_{T}(\cdot)$ and $\eta(t)$ is IBT, $h_{T}(t ; \nu)$ has either IBT or DFR shapes; see Glaser (1980). In addition, if $\lim _{t \rightarrow 0} h_{T}(t ; \nu)=0$ and $\eta(t)$ is IBT, then $h_{T}(\cdot)$ is IBT. Because $\lim _{t \rightarrow 0} h_{T}(t ; \nu)=0$ iff $\nu>2$, (R2) may be helpful to prove that $h_{T}(\cdot ; \nu)$ is IBT. In the case $\nu=2$, it may be 
useful to prove that $h_{T}(\cdot ; \nu)$ is IBT or decreasing. However, in the case $\nu=1$, as the FR has two turning points (for some values of $\alpha$ ), Glaser's result is not helpful.

As $\beta$ is a scale parameter, from now on, we take $\beta=1$, without loss of generality. Let $h_{T}(t ; \nu)$ be the BS- $t$ FR. Then, for $\nu=1, \nu=2$ and $\nu>2$, we have: $(\mathrm{R} 3) h_{T}(t ; \nu)$ is decreasing or with two turning points, for $\alpha>0.8859$, and decreasing for $\alpha>1.5964$; (R4) $h_{T}(t ; \nu)$ is decreasing or IBT for $\alpha>0.4524$, and decreasing for $\alpha>1.2649$; and (R5) $h_{T}(t ; \nu)$ has an IBT shape for $\alpha^{2}>1 / \nu$.

In order to prove these results, we use Descartes' rule of signs for a polynomial with real coefficients and with terms written in descending powers of the variable. The rule states that the number of positive roots is either equal to the number of variations in sign in the coefficients, or it is less than this number by an even integer. Next, we study the cases $\nu=1, \nu=2$, and $\nu>2$.

Case 1: $\nu=1$. From Figure 2 (left), observe that the FR decreases (i.e., it is DFR) from $\infty$ (when $t=0$ ) to zero (when $t \rightarrow \infty$ ) or has two turning points, according to the value of $\alpha$ (big values of $\alpha$ correspond to the DFR case). These turning points are solutions of $t$ in the equation $\mathrm{d} h_{T}(t ; 1) / \mathrm{d} t=0$ given by

$$
\arctan \left(\frac{t-1}{\alpha \sqrt{t}}\right)=\frac{\pi}{2}-\frac{\alpha \sqrt{t}[t+1]^{2}}{3 t^{3}+\left[\alpha^{2}+3\right] t^{2}+\left[3 \alpha^{2}-7\right] t+1} .
$$

Taking derivatives in (6) and letting $b=\alpha^{2}>0$, we obtain

$$
P(t)=3 t^{6}+[6+2 b] t^{5}+\left[-21+8 b+b^{2}\right] t^{4}+\left[12-20 b+6 b^{2}\right] t^{3}+\left[1-8 b+3 b^{2}\right] t^{2}+[-2+2 b] t+1 .
$$

We analyze the coefficients in (7), with $b>0$, in Table 1 . Then, the signs of the successive coefficients of this polynomial (from degree 6 to 0 , in this order) are given in Table 2 .

We conclude that: (i) for $\alpha>0.8859(b>[5-\sqrt{7}] / 3=0.7848)$, the BS- $t$ FR is DFR or has two turning points; (ii) for $\alpha>1.5964(b>[5+\sqrt{7}] / 3=2.5486)$, the BS- $t$ FR is DFR. Note however that, if $\alpha<0.8859$, there may still exist only two positive roots. For example, if $\alpha=0.2<0.8859$, we have only two positive roots, although the maximum number of positive roots is four (by Descartes's rule). In fact, the roots are $-3.9851,-0.4536,0.2247-0.3860 i, 0.2247+0.3860 i, 0.7848,1.1778$.

Table 1

Analysis of coefficients of the polynomial in (7).

\begin{tabular}{ccccc}
\hline Degree & Coefficient & Positive roots & Negative sign for & Positive sign for \\
\hline 6 & 3 & - & - & all $b>0$ \\
5 & $6+2 b$ & - & - & all $b>0$ \\
4 & $-21+8 b+b^{2}$ & $b=-4+\sqrt{37}=2.0827$ & $b<2.0827$ & $b>2.0827$ \\
3 & $12-20 b+6 b^{2}$ & $b=\frac{1}{3}[5 \pm \sqrt{7}]$ & $0.7848<b<2.5486$ & $b \notin] 0.7848,2.5486[$ \\
2 & $1-8 b+3 b^{2}$ & $b=\frac{1}{3}[4 \pm \sqrt{13}]$ & $0.1315<b<2.5352$ & $b \notin] 0.1315,2.5352[$ \\
1 & $-2+2 b$ & $b=1$ & $b<1$ & $b>1$ \\
0 & 1 & - & - & all $b>0$ \\
\hline
\end{tabular}

Case 2: $\nu=2$. From Figure 2 (center), we can graphically check DFR or IBT shapes (smaller values of $\alpha$ correspond to the IBT case). First, we prove that the BS- $t$ FR is $\mathrm{DFR}$ if $\alpha^{2}>8 / 5$. (Note that this condition is also equivalent to a decreasing $\mathrm{FR}$ in a 
Table 2

Signs of successive coefficients of the polynomial in (7).

\begin{tabular}{ccc}
\hline Values for $b$ & Signs of coefficients & Number of changes \\
\hline$b>2.5486$ & +++++++ & 0 \\
$2.5352<b<2.5486$ & +++-+++ & 2 \\
$2.0827<b<2.5352$ & +++--++ & 2 \\
$1<b<2.0827$ & ++---++ & 2 \\
$0.7848<b<1$ & ++----+ & 2 \\
$0.1315<b<0.7848$ & ++-+--+ & 4 \\
$b<0.1315$ & ++-++-+ & 4 \\
\hline
\end{tabular}

neighborhood of zero.) In this case

$$
h_{T}(t ; 2)=\frac{\alpha^{2}[t+1]}{\left[2 \alpha^{2} t+\{t-1\}^{2}\right]^{\frac{3}{2}}-[t-1]\left[2 \alpha^{2} t+\{t-1\}^{2}\right]} .
$$

Now, letting $y=2 \alpha^{2} t+[t-1]^{2}$, we have $\mathrm{d} h_{T}(t ; 2) / \mathrm{d} t=0$ iff

$$
y^{\frac{1}{2}}\left[y-3\{t+1\}\left\{\alpha^{2}+t-1\right\}\right]=-\left[2 t^{3}+2 \alpha^{2} t^{2}+4 \alpha^{2} t-6 t-2 \alpha^{2}+4\right] .
$$

Taking squares on both sides of (8) (note that additional solutions may then be introduced) and rearranging, we get (letting once again $b=\alpha^{2}$ )

$$
P(t)=4 t^{5}+[5 b+8] t^{4}+\left[2 b^{2}+20 b-16\right] t^{3}+\left[12 b^{2}-2 b-16\right] t^{2}+\left[18 b^{2}-44 b+28\right] t+5 b-8 .
$$

Thus, the turning points of the BS- $t$ FR are the positive roots of the polynomial in (9) (not necessarily all). We can then easily prove that if $b>8 / 5$, there are no positive roots while if $b<8 / 5$, there can exist one or three roots. (Note that $b=8 / 5$ corresponds to $\alpha=\sqrt{1.6}=1.265$.) The coefficients of the polynomial in (9) (which are quadratic functions in $b$, for $b>0$ ), its roots and its respective signs are given in Table 3. Again, following Descartes' rule, signs of the coefficients of this polynomial are given in Table 4.

Table 3

Analysis of coefficients of the polynomial in (9).

\begin{tabular}{ccccc}
\hline Degree & Coefficient & Positive roots for & Negative sign for & Positive sign for \\
\hline 5 & 4 & - & - & all $b>0$ \\
4 & $5 b+8$ & - & - & all $b>0$ \\
3 & $2\left[b^{2}+10 b-8\right]$ & $b=-5+\sqrt{33}=0.7446$ & $0<b<0.745$ & $b>0.745$ \\
2 & $2\left[6 b^{2}+-b-8\right]$ & $b=[1+\sqrt{193}] / 12=1.241$ & $0<b<1.241$ & $b>1.241$ \\
1 & $2\left[9 b^{2}-22 b+14\right]$ & no real roots & - & all $b>0$ \\
0 & $5 b-8$ & $b=\frac{8}{5}$ & $b<\frac{8}{5}$ & $b>\frac{8}{5}$ \\
\hline
\end{tabular}

If $b>1.6$, there are no positive roots (DFR case); if $1.24<b<1.6$, there is exactly one positive root (IBT case); and, if $b<1.24$, there are one or three positive roots. We can see numerically that if $b \leq 0.05$, there are indeed three positive roots (but the FR is IBT) while if $b>0.06$, there is only one positive root. However, in the case of three roots, two of them may not be solutions to the first problem. For example, if $\alpha=\sqrt{0.05}$, we have three positive roots, say $0.5230,0.6908$ and 1.1198 , but only the latter one is a solution 
Table 4

Signs of successive coefficients of the polynomial in (9).

\begin{tabular}{ccc}
\hline Values for $b$ & Signs of coefficients & Number of changes \\
\hline $0<b<0.745$ & ++--+- & 3 \\
$0.745<b<1.241$ & +++-+- & 3 \\
$1.241<b<1.6$ & +++++- & 1 \\
$b>1.6$ & ++++++ & 0 \\
\hline
\end{tabular}

to the problem. Now, using Glaser's function, we have $\eta(t)=\left[3\left\{t^{2}-1\right\}\right] /\left[2 t\left\{2 \alpha^{2} t+(t-\right.\right.$ $\left.\left.1)^{2}\right\}\right]+[t+3] /[2 t\{t+1\}]$ and thus $\eta^{\prime}(t)=0$ iff $t$ is a (positive) root of the polynomial

$$
P(t)=4 t^{6}+[4 b+8] t^{5}+\left[4 b^{2}+16 b-24\right] t^{4}+\left[24 b^{2}-44 b+8\right] t^{3}+\left[12 b^{2}-24 b+4\right] t^{2},
$$

where again $b=\alpha^{2}$. Analyzing the coefficients of the polynomial in (10), with $b>0$, in a similar way as before, we conclude that the coefficients of this polynomial have only one variation in sign for $0.2047=11 / 12-\sqrt{73} / 12<b<1+\sqrt{6} / 3=1.8165$.

According to Glaser's result, it follows that the BS- $t$ FR with $\nu=2$ is DFR or IBT if $0.2047<b<1.8165(0.4524<\alpha<1.3485)$. Combining the results obtained so far, we can finally state that the BS- $t$ FR is DFR if $b>1.6$, and it is DFR or IBT if $b>0.2047$.

Case 3: $\nu>2$. From Figure 2 (right), we suspect graphically that the BS-t FR is IBT for all $\alpha>0$ and $\nu>2$, and, as $\alpha$ increases and $\nu$ is fixed, its change point approaches zero. In this case, we have $\lim _{t \rightarrow 0} h_{T}(t ; \nu)=0$, and Glaser's theorem applies, meaning that, if $\eta(t)=-f_{T}^{\prime}(t) / f_{T}(t)$ is IBT, the BS- $t$ FR is IBT too. Now, we have $\eta(t)=$ $[\nu+1]\left[t^{2}-1\right] /\left[2\left\{\nu \alpha^{2} t+(t-1)^{2}\right\} t\right]+[t+3] /[2\{t+1\} t]$ and then $\eta^{\prime}(t)=0$ iff $t$ is a (positive) root of the polynomial (letting again $b=\alpha^{2}$ )

$$
\begin{aligned}
P(t)= & {[\nu+2] t^{6}+[2 \nu b+2 \nu+4] t^{5}+\left[\nu^{2} b^{2}+8 b \nu-3 \nu-18\right] t^{4} } \\
& +\left[-18 \nu b-2 \nu^{2} b+6 \nu^{2} b^{2}-4 \nu+16\right] t^{3} \\
& +\left[-4 \nu b-4 \nu^{2} b+3 \nu^{2} b^{2}+3 \nu-2\right] t^{2}+\left[4 \nu b-2 \nu^{2} b+2 \nu-4\right] t+2-\nu .
\end{aligned}
$$

Note that the polynomial in (11) (with $b>0$ and $\nu>2$ ) may have three positive roots. For example, for $b=0.01$ and $\nu=3$, the roots are $0.2841,0.8096$ and 1.1591. In such a case, Glaser's result is not useful (of course, the FR may still be IBT). However, we can prove that if $b>1 / \nu$, then there is only one positive root and thus the BS- $t$ FR is IBT. For $\nu>2$ and $b>0$, we analyze the coefficients of the polynomial in (11) in Table 5.

Table 5

Analysis of coefficients of the polynomial in (11).

\begin{tabular}{cccc}
\hline Degree & Coefficient & Positive roots for & Positive sign for \\
\hline 6 & $\nu+2$ & - & all $b>0$ \\
5 & $2 \nu b+2 \nu+4$ & - & all $b>0$ \\
4 & $\nu^{2} b^{2}+8 b \nu-3 \nu-18$ & $b=-\frac{4}{\nu}+\frac{1}{\nu} \sqrt{34+3 \nu}=r_{4}$ & $b>r_{4}$ \\
3 & $-18 \nu b-2 \nu^{2} b+6 \nu^{2} b^{2}-4 \nu+16$ & $b=\frac{9+\nu}{6 \nu} \pm \frac{1}{6 \nu} \sqrt{-15+42 \nu+\nu^{2}}$ & $b>r_{3}$ or $b<r_{3}^{*}$ \\
2 & $-4 \nu b-4 \nu^{2} b+3 \nu^{2} b^{2}+3 \nu-2$ & $b=\frac{2+2 \nu}{3 \nu} \pm \frac{1}{3 \nu} \sqrt{10-\nu+4 \nu^{2}}$ & $b>r_{2}$ or $b<r_{2}^{*}$ \\
1 & $4 \nu b-2 \nu^{2} b+2 \nu-4$ & $b=\frac{1}{\nu}$ or $\nu=2$ & $b<\frac{1}{\nu}=r_{1}$ \\
0 & $2-\nu$ & all $b>0$ & - \\
\hline
\end{tabular}


Let $r_{1}=1 / \nu, r_{4}=-4 / \nu+\sqrt{34+3 \nu} / \nu$ and, for $i=2,3$, let $r_{i}$ denote the positive root of the coefficient of $i$ th degree with a plus sign in its formula, and $r_{i}^{*}$ the positive root with a minus sign. We then have, for $\nu \geq 3, r_{3}^{*}<r_{2}^{*}<r_{1}<r_{4}<r_{3}<r_{2}$ (the proof is straightforward but tedious). Then, we conclude there is only one change of sign in the four situations (signs for the coefficients of degrees $6,5, \ldots, 1$, in this same order) shown in Table 6 , corresponding to the region $b>r_{1}=1 / \nu$, and this completes the proof.
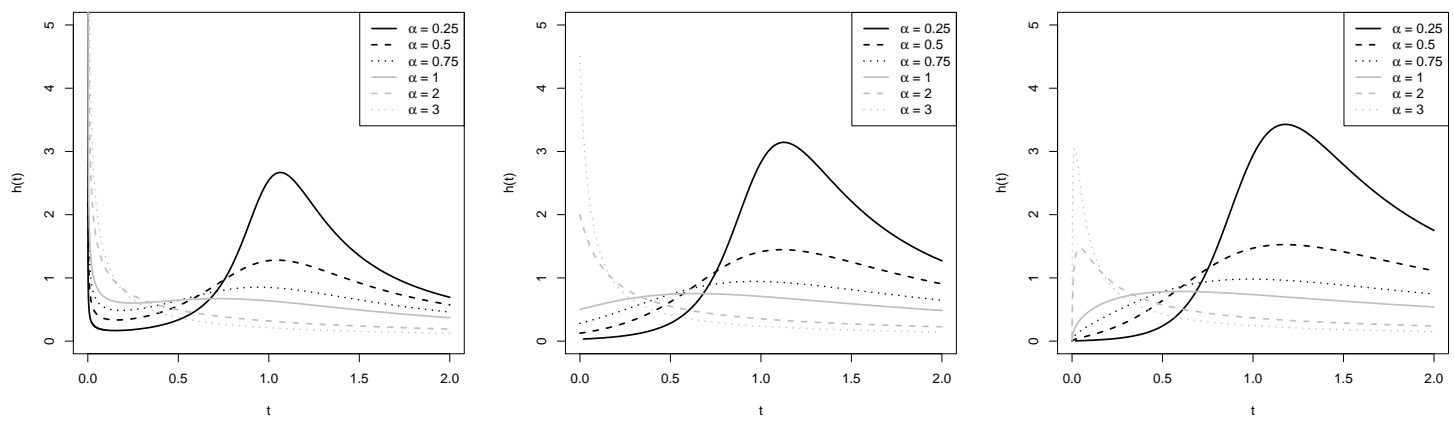

Fig. 2. BS- $t$ FR plots with $\nu=1$ (left), $\nu=2$ (center) and $\nu=3$ (right).

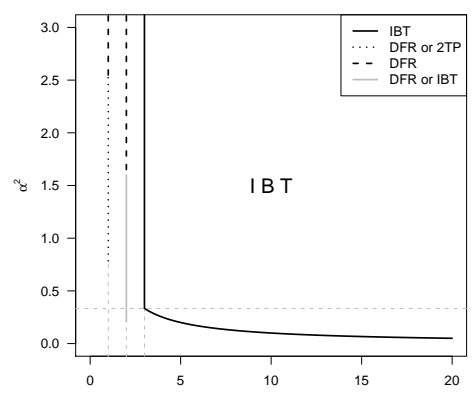

Table 6

Fig. 3. Shapes of the BS- $t$ FR.

Signs of the coefficients of the polynomial in (11).

\begin{tabular}{ccc}
\hline Values for $b$ & Signs of coefficients & Region \\
\hline$r_{1}<b<r_{4}$ & ++---- & between $r_{1}$ and $r_{4}$ \\
$r_{4}<b<r_{3}$ & +++--- & between $r_{4}$ and $r_{3}$ \\
$r_{3}<b<r_{2}$ & ++++-- & between $r_{3}$ and $r_{2}$ \\
$b>r_{2}$ & +++++- & above $r_{2}$
\end{tabular}

Letting $\nu \rightarrow \infty$, then in Case 3, we get $\alpha>0$. This is in agreement with the result for the BS distribution (see Kundu et al., 2008; Bebbington et al., 2008), which states that this distribution is IBT for all $\alpha>0$.

\section{Numerical application}

In this section, we carry out a numerical evaluation of the ML and moment estimates of the change point of the BS- $t$ FR. In addition, we analyze a real lifetime data set, estimate the change point and suggest the use of this indicator for survival analysis. Computational codes used for carrying out the simulation study and the practical application are available from the authors upon request.

\subsection{Simulation study}

Table 7 displays the ML and moment estimates of the change point $\left(t_{c}\right)$ of the BS$t$ FR. These values were obtained by using Monte Carlo simulations for the choices of 
$n=10,15,25,50$ and $\beta=1$. For every fixed $n$ and for each $\nu(\nu=4,8,15,20)$, values of $\alpha$ were estimated based on 1000 replications of $n$ pseudo-random numbers generated from the BS- $t$ distribution. For each of these 1000 estimated values of $\alpha$, the change point was calculated, by using a Newton-type method. For these lists of change points, the means and variances were calculated. By using the referred methods, $t_{c}$ point for each triplet of $n, \alpha$ and $\nu$ is presented. Table 7 shows that, when $n$ increases, the bias significantly decreases (in absolute value) for both estimation methods, as expected. The same occurs with the variance. It is also of interest to point out that when $n$ is small $(n=10,15)$, the greater variability corresponds to $\alpha=1$, although it fades out as $\nu$ increases. Also, when $n$ is moderate $(n=25,50)$, a greater variability is observed for the data with $\alpha=0.75$. Both of the estimation methods provide similar results.

\subsection{Real life example}

Here, we present an illustration with a data set analyzed earlier by Kundu et al. (2008) in the same context of the present article, but for the BS FR. The data represent the survival times of guinea pigs injected with different doses of tubercle bacilli. It is known that guinea pigs have high susceptibility to human tuberculosis. Here, we are primarily concerned with the animals in the same cage that were under the same regimen. The regimen number is the common logarithm of the number of bacillary units in $0.5 \mathrm{ml}$ of challenge solution; i.e., regimen 6.6 corresponds to $4.0 \times 106$ bacillary units per $0.5 \mathrm{ml}$ $(\log (4.0 \times 106)=6.6)$. For this regimen, 72 observations related to survival times (in days) are as follows: $12,15,22,24,24,32,32,33,34,38,38,43,44,48,52,53,54,54,55,56$, $57,58,58,59,60,60,60,60,61,62,63,65,65,67,68,70,70,72,73,75,76,76,81,83,84,85$, 87, 91, 95, 96, 98, 99, 109, 110, 121, 127, 129, 131, 143, 146, 146, 175, 175, 211, 233, 258, 258, $263,297,341,341,376$. In the sequel, these data are called survpig66.

\subsubsection{Exploratory data analysis}

Table 8 provides a descriptive summary of survpig66. Figure 8 presents (left) frequency plot by kernel estimation, (center) usual boxplot and adjusted boxplot for asymmetric data and (right) TTT plot from the survival pig data. We first conduct an exploratory data analysis (EDA) considering the following aspects. Firstly, from the results presented in Table 8 and Figure 8 (left), a positively skewed distribution -coefficient of skewness (CS) $=1.76-$ with non-negative support for survpig66 seems to be appropriate. However, a high level of kurtosis - coefficient of kurtosis $(\mathrm{CK})=5.46-$ could be an indication of a positively skewed distribution with heavy-tails. Secondly, from Figure 8 (center), apparently, some atypical data are detected by the boxplot (constructed for symmetric data). However, the adjusted boxplot (constructed for asymmetric data) shown in Figure 8 (center) does not detect atypical data on the right tail. The function adjbox of an $R$ package (www.R-project.org) called robustbase allows us to obtain the adjusted boxplot. Thus, the atypical data that we detect for survpig66 should not have a great influence on the ML estimates, which is analyzed in Subsection 4.2.2. Thirdly, from Figure 8 (right), we observe the empirical scaled TTT plot of survpig66 data. This figure indicates that a distribution with IBT-shaped FR seems to be appropriate for describing the data; see theoretical TTT plot in Figure 1. Therefore, based on the mentioned three points of the EDA, we realize a BS- $t$ distribution could be a good candidate for modeling survpig66, because it takes into account the degrees of skewness, kurtosis and variability -by the coefficient of variation $(\mathrm{CV})$ - and of the FR shape of these data. 
Table 7

Mean and variance of the moment and ML estimates of $t_{c}$ for the indicated values.

\begin{tabular}{|c|c|c|c|c|c|c|c|}
\hline \multirow[t]{2}{*}{$n$} & \multirow[t]{2}{*}{$\nu$} & \multirow[t]{2}{*}{$\alpha$} & \multirow[t]{2}{*}{$t_{c}$} & \multicolumn{2}{|c|}{ Moment estimates } & \multicolumn{2}{|c|}{ ML estimates } \\
\hline & & & & Mean & Variance & Mean & Variance \\
\hline \multirow[t]{16}{*}{10} & \multirow[t]{4}{*}{4} & 0.75 & 1.000001 & 1.070684 & 0.052519 & 0.983860 & 0.075444 \\
\hline & & 1.00 & 0.577352 & 0.833709 & 0.115734 & 0.694121 & 0.120117 \\
\hline & & 1.50 & 0.134313 & 0.420894 & 0.113441 & 0.310461 & 0.089017 \\
\hline & & 2.00 & 0.059098 & 0.213450 & 0.055438 & 0.130884 & 0.025554 \\
\hline & \multirow[t]{4}{*}{8} & 0.75 & 1.047131 & 1.138466 & 0.071230 & 1.059310 & 0.098241 \\
\hline & & 1.00 & 0.544877 & 0.789312 & 0.133016 & 0.705576 & 0.130031 \\
\hline & & 1.50 & 0.163766 & 0.344039 & 0.085710 & 0.297796 & 0.065949 \\
\hline & & 2.00 & 0.079641 & 0.174405 & 0.032410 & 0.144315 & 0.020587 \\
\hline & \multirow[t]{4}{*}{15} & 0.75 & 1.091676 & 1.267144 & 0.040012 & 1.073827 & 0.097906 \\
\hline & & 1.00 & 0.530447 & 0.723677 & 0.133682 & 0.682571 & 0.141926 \\
\hline & & 1.50 & 0.174472 & 0.330320 & 0.073934 & 0.290969 & 0.050356 \\
\hline & & 2.00 & 0.087987 & 0.160457 & 0.024863 & 0.153687 & 0.025857 \\
\hline & \multirow[t]{4}{*}{20} & 0.75 & 1.110845 & 1.144006 & 0.067805 & 1.301051 & 0.010154 \\
\hline & & 1.00 & 0.525460 & 0.670731 & 0.071870 & 0.664431 & 0.098723 \\
\hline & & 1.50 & 0.177267 & 0.332596 & 0.078415 & 0.315835 & 0.060781 \\
\hline & & 2.00 & 0.090252 & 0.158402 & 0.019418 & 0.157837 & 0.027402 \\
\hline \multirow[t]{16}{*}{15} & 4 & 0.75 & 1.000001 & 1.051977 & 0.056050 & 0.990238 & 0.053522 \\
\hline & & 1.00 & 0.577352 & 0.781092 & 0.107528 & 0.655091 & 0.099230 \\
\hline & & 1.50 & 0.134313 & 0.354774 & 0.081898 & 0.275114 & 0.052570 \\
\hline & & 2.00 & 0.059098 & 0.148404 & 0.025815 & 0.100890 & 0.012913 \\
\hline & 8 & 0.75 & 1.047131 & 1.088476 & 0.071416 & 1.053931 & 0.072337 \\
\hline & & 1.00 & 0.544877 & 0.735248 & 0.105791 & 0.693440 & 0.104247 \\
\hline & & 1.50 & 0.163766 & 0.274509 & 0.035714 & 0.244721 & 0.031524 \\
\hline & & 2.00 & 0.079641 & 0.132827 & 0.009716 & 0.115400 & 0.006828 \\
\hline & 15 & 0.75 & 1.091676 & 1.070956 & 0.092663 & 1.046344 & 0.106608 \\
\hline & & 1.00 & 0.530447 & 0.700284 & 0.096647 & 0.724091 & 0.122453 \\
\hline & & 1.50 & 0.174472 & 0.258811 & 0.029136 & 0.250958 & 0.026027 \\
\hline & & 2.00 & 0.087987 & 0.127404 & 0.007021 & 0.118265 & 0.005095 \\
\hline & 20 & 0.75 & 1.110845 & 1.145593 & 0.005903 & 1.304901 & 0.024281 \\
\hline & & 1.00 & 0.525460 & 0.483217 & 0.035508 & 0.610947 & 0.087167 \\
\hline & & 1.50 & 0.177267 & 0.269074 & 0.029891 & 0.255344 & 0.028864 \\
\hline & & 2.00 & 0.090252 & 0.131807 & 0.006936 & 0.124859 & 0.007232 \\
\hline 25 & 4 & 0.75 & 1.000001 & 1.042142 & 0.039759 & 0.990975 & 0.035333 \\
\hline & & 1.00 & 0.577352 & 0.719395 & 0.086673 & 0.634000 & 0.072789 \\
\hline & & 1.50 & 0.134313 & 0.264931 & 0.038043 & 0.200814 & 0.021566 \\
\hline & & 2.00 & 0.059098 & 0.107761 & 0.006961 & 0.080733 & 0.003557 \\
\hline & 8 & 0.75 & 1.047131 & 1.081743 & 0.050367 & 1.051924 & 0.045558 \\
\hline & & 1.00 & 0.544877 & 0.681024 & 0.072243 & 0.637277 & 0.071069 \\
\hline & & 1.50 & 0.163766 & 0.229372 & 0.017052 & 0.204343 & 0.011292 \\
\hline & & 2.00 & 0.079641 & 0.102987 & 0.002740 & 0.094846 & 0.002186 \\
\hline & 15 & 0.75 & 1.091676 & 1.064344 & 0.065308 & 1.136193 & 0.063419 \\
\hline & & 1.00 & 0.530447 & 0.630924 & 0.062990 & 0.636263 & 0.072953 \\
\hline & & 1.50 & 0.174472 & 0.224319 & 0.011978 & 0.215515 & 0.009401 \\
\hline & & 2.00 & 0.087987 & 0.111575 & 0.002354 & 0.107263 & 0.002371 \\
\hline & 20 & 0.75 & 1.110845 & 1.102231 & 0.097013 & 1.119361 & 0.073856 \\
\hline & & 1.00 & 0.525460 & 0.648222 & 0.078091 & 0.644591 & 0.068886 \\
\hline & & 1.50 & 0.177267 & 0.219861 & 0.010427 & 0.218629 & 0.010107 \\
\hline & & 2.00 & 0.090252 & 0.110894 & 0.002060 & 0.108134 & 0.001884 \\
\hline 50 & 4 & 0.75 & 1.000001 & 1.012182 & 0.035125 & 0.992639 & 0.018991 \\
\hline & & 1.00 & 0.577352 & 0.683192 & 0.056699 & 0.607537 & 0.041313 \\
\hline & & 1.50 & 0.134313 & 0.202366 & 0.014469 & 0.162919 & 0.006412 \\
\hline & & 2.00 & 0.059098 & 0.081926 & 0.001835 & 0.066743 & 0.000656 \\
\hline & 8 & 0.75 & 1.047131 & 1.056333 & 0.030301 & 1.045248 & 0.026564 \\
\hline & & 1.00 & 0.544877 & 0.619101 & 0.039423 & 0.588857 & 0.037852 \\
\hline & & 1.50 & 0.163766 & 0.195520 & 0.005237 & 0.186012 & 0.004367 \\
\hline & & 2.00 & 0.079641 & 0.092538 & 0.000842 & 0.088941 & 0.008000 \\
\hline & 15 & 0.75 & 1.091676 & 1.043809 & 0.042943 & 1.153798 & 0.020143 \\
\hline & & 1.00 & 0.530447 & 0.594042 & 0.035015 & 0.573149 & 0.032401 \\
\hline & & 1.50 & 0.174472 & 0.195229 & 0.003716 & 0.195528 & 0.003568 \\
\hline & & 2.00 & 0.087987 & 0.098666 & 0.000753 & 0.095285 & 0.000670 \\
\hline & 20 & 0.75 & 1.110845 & 1.162758 & 0.029497 & 1.172745 & 0.035136 \\
\hline & & 1.00 & 0.525460 & 0.554244 & 0.012140 & 0.595501 & 0.034005 \\
\hline & & 1.50 & 0.177267 & 0.197159 & 0.003449 & 0.195264 & 0.002980 \\
\hline & & 2.00 & 0.090252 & 0.098389 & 0.000632 & 0.098278 & 0.000647 \\
\hline
\end{tabular}


Table 8

Descriptive statistics for survpig66 data.

\begin{tabular}{cccccccccc}
\hline Median & Mean & St. Dev. & CV & CS & CK & Range & Min. & Max. & $n$ \\
\hline 70.00 & 99.82 & 81.12 & $81.27 \%$ & 1.76 & 5.46 & 364 & 12 & 376 & 72 \\
\hline
\end{tabular}
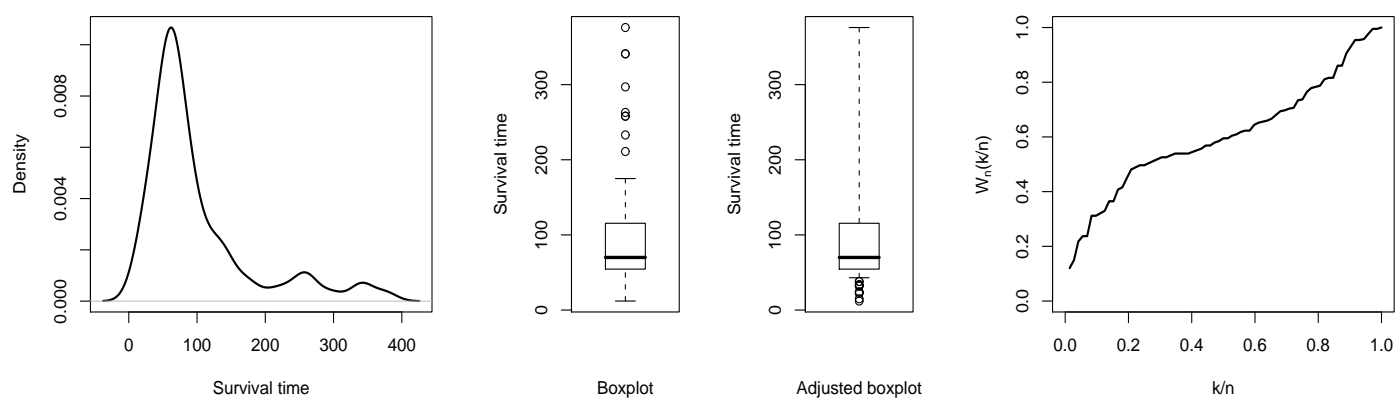

Fig. 4. (Left) PDF plot by using kernel estimation, (center) boxplot and adjusted boxplot and (right) TTT plot for survpig66 data.

\subsubsection{Estimation and checking model}

Based on survpig66, we obtain the ML estimates of $\alpha$ and $\beta$ and the optimal value of $\nu$ for the BS- $t$ distribution, which are presented in Table 9 . Figure 5 displays values of the BS- $t$ log-likelihood function against values of the parameter $\nu$ in the indicated range based on the analyzed survival pig data. This curve shows that the log-likelihood function is maximized at $\nu=5$ for survpig66.

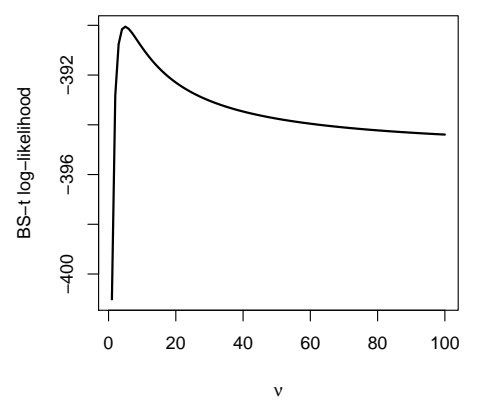

Fig. 5. Log-likelihood function of the BS- $t$ distribution against $\nu$ for survpig66 data.

Next, we carry out a brief diagnostic study based on a plot of the Mahalanobis distance (MD), which is obtained from expressions in (1) and (4) for the BS and BS- $t$ distributions, respectively. Figure 6 displays the MD for survpig66. From this figure, we identify five cases as potentially influential by using the BS distribution, which correspond to the cases $\# 1$, \#2, \#70, \#71 \#72, and just three cases by using the BS- $t$ distribution, which are $\# 1, \# 2$ and $\# 72$.

The potential influence detected by the MD is analyzed by the relative change (RC) of each parameter estimate, which is computed by dropping the influential cases and reestimating the distribution parameters by means of the expression

$$
\operatorname{RC}\left(\hat{\theta}_{1}\right)_{(i)}=\left|\frac{\hat{\theta_{1}}-\hat{\theta}_{1(i)}}{\hat{\theta_{1}}}\right| \times 100, \quad \theta_{1}=\alpha, \beta \text { or } \nu, \quad i=1, \ldots, n,
$$

where $\hat{\theta}_{1(i)}$ denotes the ML estimate of $\theta_{1}$ after the $i$ th observation is removed. We conduct a $\mathrm{RC}$ study for survpig66 and the obtained results are given in Table 9 . Based on the 

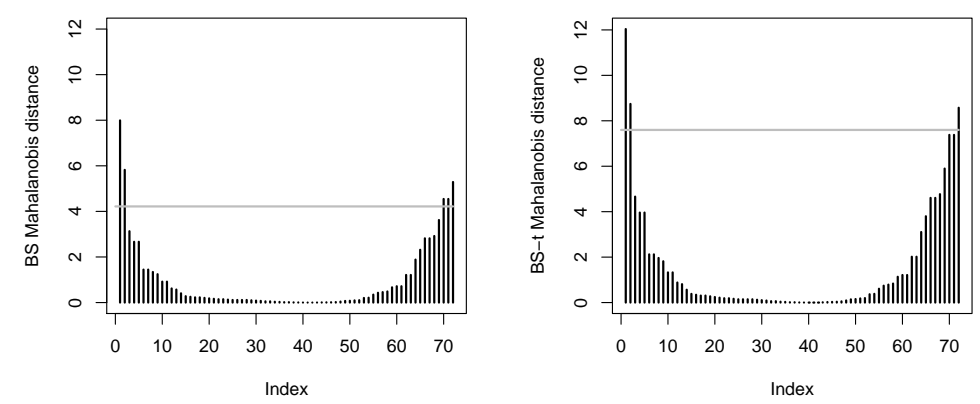

Fig. 6. MD plot based on the BS (left) and BS-t (right) models for survpig66.

RCs in this table, we do not detect a very pronounced influence of these observations on the ML estimates of $\alpha$ and $\beta$. A deeper influence study should be carried out, but we do not focus our attention on such an issue since it is beyond the scope of this work.

Table 9

ML estimates and RC (in \%) for the indicated parameter, dropped case (DC) and distribution using survpig66 data.

\begin{tabular}{ccccccccccc}
\hline \multicolumn{1}{c}{$\mathrm{BS}$} \\
$\mathrm{DC}$ & $\hat{\alpha}$ & $\mathrm{RC}$ & $\hat{\beta}$ & $\mathrm{RC}$ & $\hat{\alpha}$ & $\mathrm{RC}$ & $\hat{\beta}$ & $\mathrm{RC}$ & $\nu$ & $\mathrm{RC}$ \\
\hline- & 0.760 & - & 77.535 & - & 0.609 & - & 75.588 & - & 5 & - \\
1 & 0.721 & 5.19 & 80.410 & 3.71 & 0.631 & 3.76 & 78.076 & 3.29 & 8 & 60.00 \\
2 & 0.733 & 3.54 & 79.755 & 2.86 & 0.589 & 3.15 & 76.695 & 1.46 & 5 & 00.00 \\
70 & 0.740 & 2.60 & 75.735 & 2.32 & - & - & - & - & - & - \\
71 & 0.740 & 2.60 & 75.735 & 2.32 & - & - & - & - & - & - \\
72 & 0.736 & 3.16 & 75.518 & 2.60 & 0.560 & 7.99 & 73.748 & 2.43 & 4 & 20.00 \\
\hline
\end{tabular}

Figure 7(left) provides a probability plot with simulated envelope (bands) for the BS$t$ distribution using the survpig66 data. In this figure, we also display the estimated BS- $t$ (5) PDF (center) and the empirical CDF with estimated BS- $t$ (5) CDF (right). In these last two plots, we use the invariance property of the ML estimators for obtaining the estimated BS- $t$ (5) PDF and CDF. All of these graphical plots confirm the very good agreement between the BS-t(5) model and survpig66 data.
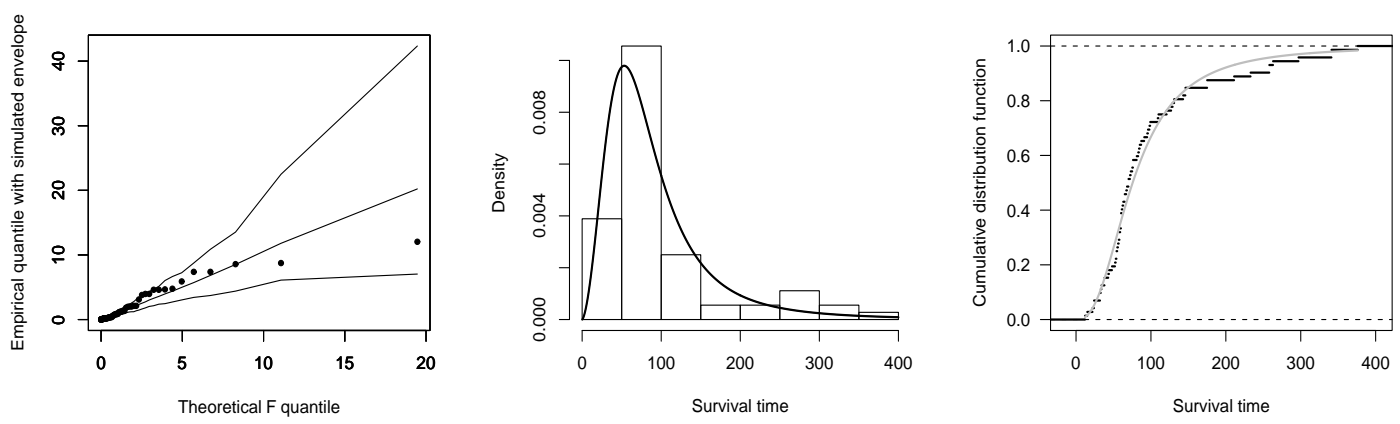

Fig. 7. Probability plot with envelope based on the BS- $t$ distribution for survpig66 data.

\subsubsection{Estimation of the BS-t FR and of its change point}

Once we find a suitable model to describe survpig66 data and estimated the model parameters, we again use the invariance property of the ML estimators for determining 
the BS- $t \mathrm{FR}$ and its change point. Let $T \sim \mathrm{BS}-t(\alpha, \beta ; \nu=5)$. Then, the $\mathrm{FR}$ of $T$ is

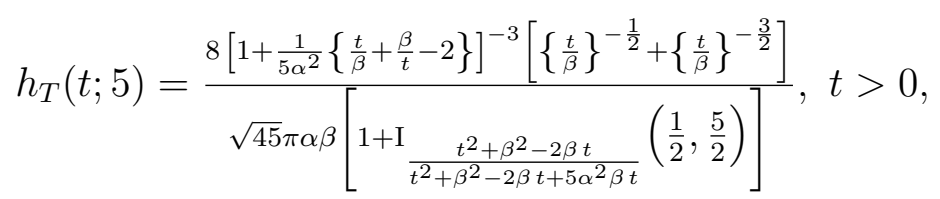

where $\mathrm{I}_{x}(\cdot, \cdot)$ is as given in (5). As mentioned earlier, the change point $\left(t_{c}\right)$ of this FR is an important value in lifetime analyses when the underlying distribution does not belong to a family with monotone FR (IFR and DFR classes), like in the case of BS distributions. Thus, when $t_{c}$ is known, we can obtain the inflection point of the FR. For $T \sim \operatorname{BS}-t(\alpha, \beta ; \nu=5)$, the change point is computed by using the ML estimates of $\hat{\alpha}$ and $\hat{\beta}$ as $\hat{t_{c}}=90.1533$ days. This value is shown in Figure 8 , where the BS- $t$ FR has been plotted using the mentioned invariance property. The value of the change point of the FR can be a useful value for practitioners. Specifically, for the problem under consideration, the value $\hat{t_{c}}=90.1533$ indicates that guinea pigs conducted by regimen 6.6 have an increasing estimated hazard, reaching its maximum at 90.1533 days, at which moment the hazard begins to decrease to zero, according to result (R1). To know this instant of the survival time can be useful for establishing a reduction in the dose of the medicine and, as a consequence, in the cost of the treatment.

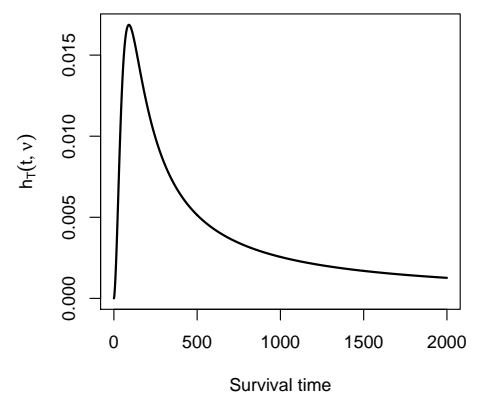

Fig. 8. Estimated curve of the BS- $t$ FR with $\nu=5$

\section{Concluding remarks and future work}

In this paper, we have carried out a shape analysis of the hazard rate of the BirnbaumSaunders-Student- $t$ distribution. Because this rate is non-monotone, its change point is a useful value for determining where the hazard begins to decrease. Such a change point can be obtained as a solution of a non-linear equation. We have provided an approximation to this change point and also shown that such an approximation works very well whenever the shape parameter is not too small. In addition, we have evaluated the performance of the maximum likelihood and moment estimates of this change point through Monte Carlo methods. We have analyzed survival times of guinea pigs and shown that the Birnbaum-Saunders-Student- $t$ distribution is quite suitable for modeling these data and the mentioned change point, allowing to illustrate some elements of the present study. To estimate this change point in terms of the survival time can be useful for establishing a reduction in the dose and, consequently, to have a smaller cost of the treatment. The authors are considering to carry out studies in this same line for cases of generalized Birnbaum-Saunders distributions with lighter tails than the classic Birnbaum-Saunders distribution. 


\section{Acknowledgements}

The authors wish to thank the Editor-in-Chief, Dr. Stanley Azen, the Associate Editor and two anonymous referees for their constructive comments on an earlier version of this manuscript. A part of this study was developed during a stay of two months of Dr. Víctor Leiva in the Department of Mathematics and Applications and the Centre of Mathematics (CMat) of the Universidade do Minho in Braga, Portugal. Research was financed by FEDER Funds through "Programa Operacional Factores de Competitividade - COMPETE" and by Portuguese Funds through FCT

- "Fundação para a Ciência e a Tecnologia", within the Project Est-C/MAT/UI0013/2011, by the CMat through the FCT Pluriannual Funding Program, Portugal, and by FONDECYT 1120879 grant, Chile.

\section{References}

Aarset, M.V., 1987. How to identify a bathtub hazard rate. IEEE Trans. Rel., 36, 106-108.

Athayde, E., Azevedo, C., Leiva, V., Sanhueza, A, 2012. About Birnbaum-Saunders distributions based on the Johnson system. Comm. Statist. Theor. Meth., 41, 2061-2079.

Balakrishnan, N., Leiva, V., López, J, 2007. Acceptance sampling plans from truncated life tests from generalized Birnbaum-Saunders distribution. Comm. Statist. Simul. Comp., 36, 643-656.

Balakrishnan, N., Leiva, V., Sanhueza, A., Cabrera, E, 2009a. Mixture inverse Gaussian distribution and its transformations, moments and applications. Statistics, 43, 91-104.

Balakrishnan, N., Leiva, V., Sanhueza, A., Vilca, F, 2009b. Scale-mixture Birnbaum-Saunders distributions: characterization and EM algorithm. Statist. Oper. Res. Trans., 33, 171-192.

Barros, M., Paula, G.A., Leiva, V, 2008. A new class of survival regression models with heavytailed errors: robustness and diagnostics. Lifetime Data Anal., 14, 316-332.

Barros, M., Paula, G.A., Leiva, V, 2009. An R implementation for generalized BirnbaumSaunders distributions. Comp. Statist. Data Anal., 53, 1511-1528.

Bebbington, M., Lai, C-D., Zitikis, R, 2008. A proof of the shape of the Birnbaum-Saunders hazard rate function. Math. Scient., 33, 49-56.

Bhatti, C.R, 2010. The Birnbaum-Saunders autoregressive conditional duration model. Math. Comp. Simul., 80, 2062-2078.

Birnbaum, Z., Saunders, S, 1969. A new family of life distributions. J. Appl. Prob., 6, 319-327.

Cancho, V.G., Louzada, F., Barriga, G.D.C, 2012. The geometric Birnbaum-Saunders regression model with cure rate. J. Statist. Plann. Infer., 142, 993-1000

Chang, D.S., Tang, L.C, 1993. Reliability bounds and critical time for the Birnbaum-Saunders distribution. IEEE Trans. Rel., 42, 464-469.

Chhikara, R.S., Folks, J.L, 1989. The Inverse Gaussian Distribution. Marcel Dekker, New York.

Cordeiro, G.M., Lemonte, A.J, 2011. The beta-Birnbaum-Saunders distribution: an improved distribution for fatigue life modeling. Comp. Statist. Data Anal., 55, 1445-1461.

Cysneiros, A.H.M.A., Cribari-Neto, F., Araújo, C.A.G, 2008. On Birnbaum-Saunders inference. Comp. Statist. Data Anal., 52, 4939-4950.

Díaz-García, J.A., Leiva, V, 2005. A new family of life distributions based on elliptically contoured distributions. J. Statist. Plann. Infer., 128, 445-457 (Erratum: J. Statist. Plann. Infer., $137,1512-1513)$

Glaser, R.A, 1980. Bathtub and related failure rate characterizations. J. Amer. Statist. Assoc. , 75, 667-672.

Guiraud, P., Leiva, V., Fierro, R, 2009. A non-central version of the Birnbaum-Saunders distribution for reliability analysis. IEEE Trans. Rel., 58, 152-160.

Gupta, R.C., Kannan, N., Raychoudhuri, A, 1997. Analysis of log-normal survival data. Math. Biosci., 139,103-115. 
Johnson, N.L., Kotz, S., Balakrishnan, N, 1995. Continuous Univariate Distributions-Vol. 2. Second edition. John Wiley and Sons, New York.

Kotz, S., Leiva, V., Sanhueza, A, 2010. Two new mixture models related to the inverse Gaussian distribution. Meth. Comp. Appl. Prob., 12, 199-212.

Kundu, D., Kannan, N., Balakrishnan, N, 2008. On the hazard function of Birnbaum-Saunders distribution and associated inference. Comp. Statist. Data Anal., 52, 2692-2702.

Lawless, J.F, 2002. Statistical Models and Methods for Lifetime Data. Second edition. John Wiley and Sons, New York.

Leiva, V., Barros, M., Paula, G.A., Galea, M., 2007. Influence diagnostics in log-BirnbaumSaunders regression models with censored data. Comp. Statist. Data Anal., 51, 5694-5707.

Leiva, V., Riquelme, M., Balakrishnan, N., Sanhueza, A, 2008a. Lifetime analysis based on the generalized Birnbaum-Saunders distribution. Comp. Statist. Data Anal., 52, 2079-2097.

Leiva, V., Barros, M., Paula, G., Sanhueza, D, 2008b. Generalized Birnbaum-Saunders distributions applied to air pollutant concentration. Environmetrics, 19, 235-249.

Leiva, V., Sanhueza, A., Angulo, J.M, 2009. A length-biased version of the Birnbaum-Saunders distribution with application in water quality. Stoch. Environ. Res. Risk. Assess., 23, 299-307.

Leiva, V., Vilca, F., Balakrishnan, N., Sanhueza, A, 2010a. A skewed sinh-normal distribution and its properties and application to air pollution. Comm. Statist. Theor. Meth., 39, 426-443.

Leiva, V., Sanhueza, A. Kotz, S., Araneda, N, 2010b. A unified mixture model based on the inverse gaussian distribution. Pak. J. Statist., 26, 445-460.

Leiva, V., Athayde, E., Azevedo, C., Marchant, C., 2011a. Modeling wind energy flux by Birnbaum-Saunders distribution with unknown shift parameter. J. Appl. Statist., 38, 28192838.

Leiva, V., Soto, G., Cabrera, E., Cabrera, G, 2011b. New control charts based on the BirnbaumSaunders distribution and their implementation. Col. J. Statist., 34, 147-176.

Marshall, A.W., Olkin, I, 2007. Life Distributions. Springer, New York.

Nelson, W.B, 1990. Accelerated Testing: Statistical Models, Test Plans, and Data Analysis. John Wiley and Sons, New York.

Paula, G.A., Leiva, V., Barros, M., Liu, S, 2012. Robust statistical modeling using the BirnbaumSaunders-t distribution applied to insurance. Appl. Stoch. Mod. Bus. Ind., 28, 16-34.

Sanhueza, A., Leiva, V., Balakrishnan, N, 2008. The generalized Birnbaum-Saunders distribution and its theory, methodology and application. Comm. Statist. Theor. Meth., 37, 645-670.

Saunders, S.C, 2007. Reliability, Life Testing and Prediction of Services Lives. Springer, New York.

Vanegas, L.H., Rondona, L.M., Cysneiros, F.J.A, 2012. Diagnostic procedures in BirnbaumSaunders nonlinear regression models. Comp. Statist. Data Anal., 56, 1662-1680.

Vilca, F., Leiva, V, 2006. A new fatigue life model based on the family of skew-elliptical distributions. Comm. Statist. Theor. Meth., 35, 229-244.

Vilca, F., Sanhueza, A., Leiva, V., Christakos, G., 2010. An extended Birnbaum-Saunders model and its application in the study of environmental quality in Santiago, Chile. Stoch. Envir. Res Risk Ass., 24, 771-782.

Vilca, F., Santana, L., Leiva, V., Balakrishnan, N, 2011. Estimation of extreme percentiles in Birnbaum-Saunders distributions. Comp. Statist. Data Anal., 55, 1665-1678. 